\title{
Beiträge zur Kenntnis der Cynipiden (Hym.).
}

Von H. Hedicke, Berlin-Steglitz.

IV.

\section{Über einige Cynipiden des Dentschen Entomologischen Museums.}

Die Untersuchung der mir vom Deutschen Entomologischen Museum zur Bearbeitung übergebenen Cynipiden ergab außer der Auffindung zweier für die mitteleuropäische Fauna neuer Tiere eine neue Gattung mit zwei Arten schlesischer Provenienz, eine neue Art aus Sardinien und das bisher unbekannte Männchen einer weiteren Art.

\section{Aspicerinae.}

Aspicera brevispina Kieff. - Ein đ von Konow in Mecklenburg gesammelt, bisher nur von Barcelona, Spanien, bekannt.

Omalaspis norica Gir. \& nov. Vom o durch dio 14 gliedrigen Antennen unterschieden, bei denen die zwei Basalglieder pechbraun, die übrigen rotbraun sind. - Von Konow am 13. Mai 1896 in Mecklenburg gesammelt, bisher nur bei Graz, Österreich, gefunden.

Omalaspoides nov. gen. - Kopf so breit wie der Thorax, Hinterkopf lederartig gerunzelt; Augen behaart; Antenne beim $\delta$ fadenförmig, Flagellenglieder langgestreckt, walzenförmig, beim 우 perlschnurförmig, mittlere Flagellenglieder fast kugelig; Mesonotum und Scutellum ungekielt, Parapsidenfurchen tief und durchlaufend, zwischen ihnen in der vorderen Hälfte des Mesonotums zwei kurze, schwache Längsfurchen; Scutellum ohne Dorn, vorn mit zwei tiefen Gruben, Scheibe stark unregelmäßig gerunzelt; Radialzelle geschlossen; Abdominalstiel ringförmig, gefurcht.

Der Gattung Omalaspis Gir. am nächsten stehend, von dieser aber durch den Bau der Fühler des o scharf getrennt.

Typus: Omalaspoides letzneri nov. spec. - Schwarz; Kopf und Thorax mit gelblich grauen Haaren zerstreut besetzt; Kopf unregelmäßig lederartig gerunzelt, beim of stärker als beim $\delta$, über den rotbraunen Mandibeln eine glatte, etwas erhabene Stelle. Antenne beim ơ so lang wie der Körper, 1. Glied zweimal so lang wie dick, 2. kugelig, 3. viermal so lang wie dick, 4. bis 13. Glied gleich grob, etwa dreimal so lang wie dick, 14. etwas länger; Antenne beim o kürzer als Kopf und Thorax zusammen, 1. Glied dreimal so lang wie dick, 2. kugelig, 3. so lang wie das erste, 4. zweimal so lang wie dick, 5. bis 12. Glied gleich groß, kaum länger als breit, Endglied fast zweimal so lang wie dick. Prothorax senkrecht ab- 
gestutzt, am oberen Rande schwach bogig ausgerandet; Propleuren in der dorsalen Hälfte mit in Längsreihen angeordneten Punkten, in der ventralen längsgerunzelt; Mesonotum glänzend, sehr zerstreut punktiert; Parapsidenfurchen tief, nach hinten verbreitert, zwischen ihnen im hinteren Drittel eine kurze, breite Längsfurche. Scutellum vom Mesonotum durch eine tiefe Querfurche getrennt, von oben gesehen trapezförmig; der ganze Thorax beim o s stärker skulptiert als beim $\delta$. Flügel gänzlich unbehaart, Cubitalis erloschen; 2. Abschnitt der Radialis und 4. Abschnitt der Subcostalis fast farblos; Beine gelbrot bis auf die Coxen und die proximalen Enden der Femora, die schwarz gefärbt sind. Abdomen beim of kaum so lang, beim \& länger als der übrige Körper, schwarz, beim of unterseits rötlich braun, 2. Tergit in der proximalen Hälfte längsgefurcht; Ende des Abdomens beim $\delta$ abgerundet, beim 우 zugespitzt.

Länge: $\delta 4 \mathrm{~mm}$, 우 $5,2 \mathrm{~mm}$.

Ein $\delta$, ein $q$ von Letzner in Schlesien gesammelt und ihm $\mathrm{zu}$ Ehren benannt.

Omalaspoides silesiacus nov. spec.

§. - Von O. letzneri m. durch folgende Merkmale zu unterscheiden :

Antennen bis auf die beiden pechschwarzen Basalglieder dunkel gelbbraun; Kopf glatt, zerstreut punktiert, Skulptur des Thorax wie bei letzneri, aber weit schwächer; Flügel behaart, Radialis und Subcostalis in allen Abschnitten gelbbraun gefärbt. Zweites Abdominaltergit fast so lang wie das dritte, im proximalen Drittel gelblich grau behaart.

Länge: $4 \mathrm{~mm}$.

Ein $\delta$ von Letzner in Schlesien gesammelt.

\section{Eucoilinae.}

Rhoptromeris afra Kieff. - Ein ơ von Konow in Fürstenberg i. M., 26. August 1889, gesammelt; bisher nur von Tenerifa bekannt.

Chrestosema laeviusculum nov. spec.

§. - Schwarz; Kopf rundlich, glatt, glänzend, Augen am Innenrand gelbbraun, zwischen ihnen und den Antennen je eine kurze, tiefe Furche, Antenne schwarz, drittes Glied am längsten, viermal so lang wie breit. Thorax glatt, fast unskulptiert, Seitenfurchen des Mesonotums breit und tief, Mittellängsfurchen schwach angedeutet; Scutellum vom Mesonotum durch zwei tiefe Gruben 
getrennt. Mediansegment fast senkrecht, mit zwei bogigen Längsleisten; Metapleuren glatt, über den Coxen mit einer flachen, napfförmigen Vertiefung. Flügel glashell, behaart und bewimpert, Adern gelbbraun, zweiter Abschnitt der Radialis länger als der erste, dieser nach innen gebogen; Femora an beiden Enden, Tibien und Tarsen rotbraun, Coxen, Trochanteren und Mitte der Femora schwarz. Abdomen so lang wie der Thorax, schwarz, glänzend.

Länge: $2,5 \mathrm{~mm}$.

Ein ơ von Krauße bei Asuni, Sardinien, gefangen.

\section{Bemerkungen zum „Psyllidarum Catalogus“ von 6. Aulmann. Von Dr. Friedrich Zacher, Berlin-Dahlem.}

Vor kurzem ist im Verlage von W. Junk ein von G. Aulmann verfaßtes „synonymisch“-biologisches Verzeichnis der Blattflöhe erschienen unter dem Titel „Psyllidarum Catalogus“. Soweit ich den Katalog an der Hand der mir gerade augenblicklich zur Verfügung stehenden Literatur nachprüfen konnte, vermisse ich darin, unter Berücksichtigung der bis Ende 1911 erschienenen Arbeiten, den Nachweis der Gattungen:

Allotrioza Crawf. Pomona J. of Entom. III, p. 442, Februar 1911.

Epitrioza Crawf. Pomona J. of Entom. III, p. 452. Dieser

Name ist synonym zu Kuwayama Crawf. l. c., p. 503.

Epicarsa Crawf. l. c. III, p. 488, Mai 1911.

Neotrioza Crawf. 1. c. III, p. 450. Dieser Name ist synonym zu Neotriozella Crawf. l. c. III, p. 503.

Paratrioza Crawf. l. c. II, p. 229, Mai 1910.

Phylloplecta für Trioza tripunctata Fitch, cf. Riley, 1883.

Triozoidea Crawf. l. c. III, p. 491, Mai 1911.

Ferner fehlt ein neuer Gattungsname:

Cardiaspina Crawt. pro Cardiaspis Schwarz 1. c. III, p. 632, Dezember 1911.

Nicht auffinden konnte ich die Beschreibung einer bei Aulmann fehlenden Gattung:

Psyllopa cf. Crawf. 1. c. III, p. 628.

Des weiteren fehlen folgende Arten und Varietäten:

Aphalara communis Crawf. 1. c. II, p. 499. Colorado, Arizona, California, Nevada, Wisconsin, Louisiana, Alabama, Wyoming. 


\section{$2 \mathrm{BHL}$ Biodiversity Heritage Library}

1913. "Beitrage zur Kenntnis der Cynipiden. iv." Entomologische Mitteilungen 2, 146-148. https://doi.org/10.5962/bhl.part.14991.

View This Item Online: $\underline{\text { https://www.biodiversitylibrary.org/item/38257 }}$

DOI: https://doi.org/10.5962/bhl.part.14991

Permalink: https://www.biodiversitylibrary.org/partpdf/14991

\section{Holding Institution}

Smithsonian Libraries

\section{Sponsored by}

Smithsonian

\section{Copyright \& Reuse}

Copyright Status: NOT_IN_COPYRIGHT

This document was created from content at the Biodiversity Heritage Library, the world's largest open access digital library for biodiversity literature and archives. Visit BHL at https://www.biodiversitylibrary.org. 